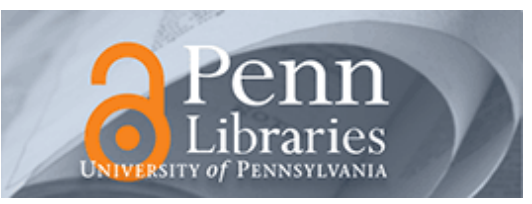

University of Pennsylvania

ScholarlyCommons

October 2004

\title{
Achieving Proportional Fairness Using Local Information in Aloha Networks
}

\author{
Koushik Kar \\ Rensselaer Polytechnic Institute \\ Saswati Sarkar \\ University of Pennsylvania, swati@seas.upenn.edu \\ Leandros Tassiulas \\ University of Thessaly
}

Follow this and additional works at: https://repository.upenn.edu/ese_papers

\section{Recommended Citation}

Koushik Kar, Saswati Sarkar, and Leandros Tassiulas, "Achieving Proportional Fairness Using Local Information in Aloha Networks", . October 2004.

Copyright 2004 IEEE. Reprinted from IEEE Transactions on Automatic Control, Volume 49, Issue 10, October 2004, pages 1858-1862.

Publisher URL: http://ieeexplore.ieee.org/xpl/tocresult.jsp?isNumber=29559\&page=2

This material is posted here with permission of the IEEE. Such permission of the IEEE does not in any way imply IEEE endorsement of any of the University of Pennsylvania's products or services. Internal or personal use of this material is permitted. However, permission to reprint/republish this material for advertising or promotional purposes or for creating new collective works for resale or redistribution must be obtained from the IEEE by writing to pubs-permissions@ieee.org. By choosing to view this document, you agree to all provisions of the copyright laws protecting it.

This paper is posted at ScholarlyCommons. https://repository.upenn.edu/ese_papers/66

For more information, please contact repository@pobox.upenn.edu. 


\title{
Achieving Proportional Fairness Using Local Information in Aloha Networks
}

\author{
Abstract \\ We address the problem of attaining proportionally fair rates using Aloha protocols at the medium access \\ layer. We consider a wireless network where all nodes need not be in transmission ranges of each other. \\ We show how the attempt probabilities in Aloha protocols should be set so that the achieved rates are \\ globally proportionally fair. For both slotted and unslotted Aloha,we argue that each node can compute its \\ optimal attempt probability just by knowing some minimal information about the network topology in its \\ two-hop radius. \\ Keywords \\ Aloha networks, fairness, local information \\ Comments \\ Copyright 2004 IEEE. Reprinted from IEEE Transactions on Automatic Control, Volume 49, Issue 10, \\ October 2004, pages 1858-1862. \\ Publisher URL: http://ieeexplore.ieee.org/xpl/tocresult.jsp?isNumber=29559\&page=2 \\ This material is posted here with permission of the IEEE. Such permission of the IEEE does not in any way \\ imply IEEE endorsement of any of the University of Pennsylvania's products or services. Internal or \\ personal use of this material is permitted. However, permission to reprint/republish this material for \\ advertising or promotional purposes or for creating new collective works for resale or redistribution must \\ be obtained from the IEEE by writing to pubs-permissions@ieee.org. By choosing to view this document, \\ you agree to all provisions of the copyright laws protecting it.
}


[4] R. A. Freeman and P. V. Kokotovic, Robust Nonlinear Control Design: State Space and Lyapunov Techniques. Boston, MA: Birkhäuser, 1996.

[5] S. Gutman, "Uncertain dynamical systems-A Lyapunov min-max approach," IEEE Trans. Automat. Contr, vol. AC-24, pp. 437-443, Mar. 1979.

[6] A. Isidori, Nonlinear Control Systems I \& II. New York: SpringerVerlag, 1995 and 1999.

[7] H. Khalil, Nonlinear Systems, 2nd ed. Upper Saddle River, NJ: Prentice-Hall, 1996.

[8] K. H. Khalil and F. Esfandiari, "Semiglobal stabilization of a class of nonlinear systems using output feedback," IEEE Trans. Automat. Contr., vol. 38, pp. 1412-1415, Sept. 1993.

[9] M. Krstic, I. Kanellakppoulos, and P. V. Kokotovic, Nonlinear and Adaptive Control Design. New York: Wiley, 1995.

[10] Z. Qu, "Global stabilization of nonlinear systems with a class of unmatched uncertainties," Syst. Control Lett., vol. 18, pp. 301-307, 1992.

[11] _ , "Robust control of nonlinear uncertain systems under generalized matching conditions," Automatica, vol. 29, pp. 985-998, 1993.

[12] - Robust Control of Nonlinear Uncertain Systems. New York: Wiley, 1998.

[13] — , "Robust control of nonlinear systems by estimating time variant uncertainties," IEEE Trans. Automat. Contr., vol. 47, pp. 115-121, Jan. 2002.

[14] - "Adaptive and robust controls of uncertain systems with nonlinear parameterization," in Proc. American Control Conf., Anchorage, AK, May 2002, pp. 1791-1796.

[15] Z. Qu and Y. Jin, "Robust control of nonlinear systems in the presence of unknown exogenous dynamics," in Proc. 2001 IEEE Conf. Decision and Control, Orlando, FL, Dec. 2001, pp. 2784-2790.

[16] S. Sastry and M. Bodson, Adaptive Control: Stability, Convergence, and Robustness. Upper Saddle River, NJ: Prentice-Hall, 1989.

[17] A. Saengdeejing and Z. Qu, "Simplified robust control for nonlinear uncertain systems: A method of projection and on-line estimation," in Proc. Amer. Control Conf., Anchorage, AK, May 2002, pp. 3425-3430.

[18] R. Sepulchre, M. Jankovic, and P. V. Kokotovic, Constructive Nonlinear Control. New York: Springer-Verlag, 1997.

[19] E. D. Sontag and Y. Wang, "On characterizations of the input-to-state stability property," Syst. Control Lett., vol. 24, pp. 351-359, 1995.

[20] J. Tsinias, "Observer design for nonlinear systems," Syst. Control Lett., vol. 42, pp. 233-244, 1989.

\section{Achieving Proportional Fairness Using Local Information in Aloha Networks}

Koushik Kar, Saswati Sarkar, and Leandros Tassiulas

\begin{abstract}
We address the problem of attaining proportionally fair rates using Aloha protocols at the medium access layer. We consider a wireless network where all nodes need not be in transmission ranges of each other. We show how the attempt probabilities in Aloha protocols should be set so that the achieved rates are globally proportionally fair. For both slotted and unslotted Aloha, we argue that each node can compute its optimal attempt probability just by knowing some minimal information about the network topology in its two-hop radius.
\end{abstract}

Index Terms-Aloha networks, fairness, local information.

\section{INTRODUCTION}

Medium access control (MAC) algorithms are used in wireless networks to control access to a shared wireless medium, and thereby reduce collisions, ensure high system throughput, and distribute the available bandwidth fairly among the competing streams of traffic. We address the issue of designing medium access protocols for attaining proportionally fair rates [2] in wireless networks. The problem of designing distributed access control for attaining fair rates in wireless networks has not been adequately addressed. Tassiulas et al. [7] have proposed a centralized algorithm for attaining max-min fairness in certain classes of networks. However, centralized strategies can not be used in large, dynamic ad-hoc networks. In another line of work, Nandagopal et al. [5] and Ozugur et al. [6] have proposed decentralized heuristic medium access strategies that try to achieve some fairness objectives, but the authors did not prove the fairness properties of these approaches.

The problem of fair rate control at the transport layer of wired networks has however been extensively researched, e.g., [3] and [4]. In this context, researchers have shown that globally fair rates can be attained via distributed approaches based on convex programming. However, these techniques can not be directly applied to wireless networks. This is because the rates attained by most wireless MAC protocols can only be indirectly controlled by regulating the transmission probabilities or back-off window sizes. It is difficult to attain the globally fair rates in wireless networks through a distributed approach as the feasible rate region is a complex, nonconvex, and nonseparable function of the attempt probabilities or back-off window sizes. In contrast, distributed rate control algorithms have been developed for wired networks, using the feature that the feasible rate region can be represented by a set of simple, separable, convex constraints.

Manuscript received December 10, 2003; revised June 21, 2004. Recommended by Associate Editor R. Srikant. The work of K. Kar was supported by the National Science Foundation under Grant A11415.2260. The work of S. Sarkar was supported by the National Science Foundation under Grants ANI01-06984 and NCR02-38340.

K. Kar is with the Department of Electrical, Computer, and Systems Engineering, Rensselaer Polytechnic Institute, Troy, NY 12180 USA (e-mail: koushik@ecse.rpi.edu).

S. Sarkar is with the Department of Electrical Engineering, University of Pennsylvania, Philadelphia, PA 19104 USA (e-mail: swati@ee.upenn.edu).

L. Tassiulas is with the Computer Engineering and Telecommunications Department, University of Thessaly, 38221 Volos, Greece (e-mail: leandros@inf.uth.gr).

Digital Object Identifier 10.1109/TAC.2004.835596 
Since a wireless channel is shared by several users, distributed MAC protocols typically result in some loss of bandwidth due to collisions. This bandwidth loss depends on the access protocol. Thus, the feasible rate region depends on the protocol, and the feasible rate region for any distributed protocol is a subset of that for the optimal centralized algorithm. Therefore, the fair access control problem must be considered in the context of specific protocols. We study the fair access control problem for Aloha protocols [1]. For both slotted and unslotted Aloha, we show that globally proportionally fair rates can be attained if each node selects its attempt probability appropriately. A node can compute its optimal attempt probability by knowing some minimal information about the network topology in its two-hop neighborhood.

We consider proportional fairness as this notion has certain nice fairness properties [2]. Moreover, in Aloha networks, the optimization problem for attaining proportional fairness is convex and separable, which allows us to develop computationally simple algorithms for attaining the optimal rates. The extension of our results to other fairness metrics remains an open question. The fair access control problem for more complex protocols like carrier sense multiple access (CSMA) also remains an open question. We believe that our results for attaining proportionally fair rates using Aloha would provide insights for designing the optimal access control strategies for other protocols and other fairness metrics.

We consider fair allocation of bandwidth at the MAC layer. Since the MAC layer is associated with packet delivery over a single link (or hop), we consider the fairness issues only at a link level. The network and transport layers are associated with end-to-end packet delivery and, therefore, end-to-end fairness questions would involve these higher layers as well. Fair allocation of end-to-end bandwidth will require cross-layer optimizations involving the network and MAC layers, or the transport, network, and MAC layers. To achieve this, several different research problems must be solved: 1) optimizing the attempt rates at each hop for multihop sessions, 2) appropriately deciding the routes, and 3) optimizing the parameters of the transport layer protocols. These are beyond the scope of this note. However, the analytical framework we develop for MAC layer fairness provides the first step in that direction.

The note is organized as follows. In Section II, we describe our system model. In Sections III and IV, we show how to attain proportionally fair rates for slotted and unslotted aloha protocols respectively. We present all proofs in the Appendix.

\section{SYSTEM MODEL}

We consider a general wireless network, where all nodes need not be in transmission ranges of each other. For simplicity, we assume symmetric transmission, i.e., a node $i$ can receive signal from a node $j$ if and only if node $j$ can receive signal from node $i$. However, our analysis can be generalized to the case when this assumption does not hold. Now, a wireless network can be modeled as an undirected graph $G=(N, L)$, where $N$ and $L$ respectively denote the set of nodes and the set of undirected links, and a link exists between two nodes if and only if they can receive each other's signals. A directed edge $(i, j)$ represents an active communication pair, and $E$ is the set of directed edges. Note that there are $2|L|$ possible communication pairs, but only a few pairs may be actively communicating. Every edge $(i, j) \in E$ is always backlogged, i.e., node $i$ always has packets to send to node $j$. Each node $i$ is involved in at least one active communication, i.e., there exists some $j$ such that either $(i, j)$ or $(j, i)$ is in $E$. Without loss of generality, capacity of each channel is 1 unit.

For any node $i$, the set of $i$ 's neighbors, $K_{i}=\{j:(i, j) \in L\}$, represents the set of nodes that can receive $i$ 's signals. For any node $i$, the set of out-neighbors of $i, O_{i}=\{j:(i, j) \in E\} \subseteq K_{i}$, represents the set of neighbors to which $i$ is sending traffic. Also, for any node $i$, the set of in-neighbors of $i, I_{i}=\{j:(j, i) \in E\} \subseteq K_{i}$, represents the set of neighbors from which $i$ is receiving traffic. A transmission from node $i$ reaches all of $i$ 's neighbors. Each node has a single transceiver. Thus, a node can not transmit and receive simultaneously. We do not assume any capture, i.e., node $j$ can not receive any packet successfully if more than one of its neighbors are transmitting simultaneously. Therefore, a transmission on edge $(i, j) \in E$ is successful if and only if no node in $K_{j} \cup\{j\} \backslash\{i\}$ transmits during the transmission on $(i, j)$.

\section{FAIR MAC IN SLOTted AlohA}

We first formulate the fair rate allocation problem for slotted Aloha as an optimization problem, and then provide a policy that achieves globally fair rates using only local information.

\section{A. Problem Formulation}

In slotted Aloha, time is divided into fixed-length slots, the length of a slot being equal to the transmission time of a packet. We first derive the throughput expressions in slotted Aloha. In each slot, each node $i$ transmits a packet with probability $P_{i}$. If $i$ does not have an outgoing edge, i.e., $O_{i}=\phi$, then $P_{i}=0$. Once $i$ decides to transmit in a slot, it selects a destination $j \in O_{i}$ with probability $p_{(i, j)} / P_{i}$, where $\sum_{j \in O_{i}} p_{(i, j)}=P_{i}$. Therefore, in each slot, a packet is transmitted on edge $(i, j)$ with probability $p_{(i, j)}$. Let $\underline{p}=\left(p_{e}, e \in E\right)$ be the vector of transmission probabilities on all edges. Then, the throughput or the successful data rate on edge $e=(i, j), x_{e}$, is (see [1, Sec. 4.6.2])

$$
x_{(i, j)}(\underline{p})=p_{(i, j)}\left(1-P_{j}\right) \prod_{k \in K_{j} \backslash\{i\}}\left(1-P_{k}\right) .
$$

In the previous expression, if $O_{k}=\phi$ for any $k \in N$, then the term $P_{k}$ should be interpreted as zero. In (1), the term $\left(1-P_{j}\right) \prod_{k \in K_{j} \backslash\{i\}}\left(1-P_{k}\right)$ is the probability that a packet transmitted on edge $(i, j)$ is successfully received at $j$. The attempt probability vector, $\underline{p}^{*}=\left(p_{e}^{*}, e \in E\right)$, that attains proportionally fair rates is given by

$$
\underline{p}^{*}=\arg \max _{0 \leq p_{e} \leq 1, e \in E} \sum_{e \in E} \log \left(x_{e}(\underline{p})\right)
$$

where the $x_{e}(p)$ are given by (1). As in the rest of this note, the $\log$ function in the aforementioned expression is a natural logarithm.

\section{B. Optimal Attempt Probabilities}

Theorem 1: The optimal attempt probability on any edge $(i, j) \in$ $E, p_{(i, j)}^{*}$, as defined by (2), is given by

$$
p_{(i, j)}^{*}=\frac{1}{\left|I_{i}\right|+\sum_{k \in K_{i}}\left|I_{k}\right|} .
$$

Clearly, $p_{(i, j)}^{*} \geq 0, \forall(i, j) \in E$. Note that $O_{i} \subseteq K_{i}$. Thus, $\sum_{k \in K_{i}}\left|I_{k}\right| \geq \sum_{k \in O_{i}}\left|I_{k}\right| \geq\left|O_{i}\right|$. Since $(i, j) \in E, O_{i} \neq \phi$. Thus, $\left|O_{i}\right| \geq 1$. Therefore, $\sum_{k \in K_{i}}\left|I_{k}\right| \geq 1$, implying $p_{(i, j)}^{*} \leq$ $1, \forall(i, j) \in E$. Also, the optimum transmission probability for each 


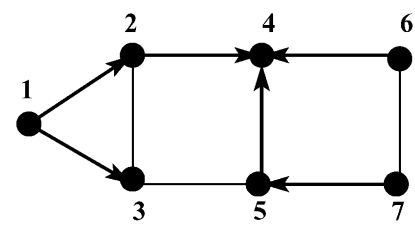

Fig. 1. Example network. (Nodes 1 and 7 belong to $N^{\prime}$, and all other nodes belong to $N \backslash N^{\prime}$.) The bold lines represent the (directed) edges, and the other lines represent the (undirected) links where there are no edges.

node $i, P_{i}^{*}=\sum_{j:(i, j) \in E} p_{(i, j)}^{*}=\left|O_{i}\right| /\left(\left|I_{i}\right|+\sum_{k \in K_{i}}\left|I_{k}\right|\right)$. Clearly, $P_{i}^{*} \geq 0$ for each node $i$. Since $\sum_{k \in K_{i}}\left|I_{k}\right| \geq\left|O_{i}\right|, P_{i}^{*} \leq 1$. Thus, $P_{i}^{*}$ and $p_{(i, j)}^{*}$ are valid probability measures.

A node can decide its optimal attempt probability only if it knows the number of its in-neighbors and the number of its neighbors' in-neighbors. A node can determine the latter as follows. When the network is formed, or when the network topology changes due to the joining, leaving, or movement of nodes, each node broadcasts the number of its in-neighbors to all nodes in its transmission range. This incurs a small additional information exchange.

Now, consider the special case in which all nodes are in each other's transmission range, and therefore, at most one node can send data successfully at any time. Let $n$ nodes send packets to a single destination node. From Theorem 1, the optimal attempt probabilities in each of the $n$ edges is $(1 / n)$, and from (1), the system throughput for proportionally fair rates is $(1-(1 / n))^{(n-1)}$. As $n$ approaches $\infty$, the system throughput for proportionally fair rates becomes $(1 / e) \approx 37 \%$, which equals the maximum system throughput attained for any choice of attempt probabilities in slotted Aloha in this network [1].

\section{FAIR MAC IN UNSLOTTED ALOHA}

We first state the assumptions and derive the throughput expressions. We then show how the optimal attempt rates can be computed using local information.

\section{A. Problem Formulation}

In unslotted Aloha, there are no slots, and a node can transmit a packet at any time. We assume that each packet has a fixed transmission time, $T$. We assume that transmission attempts by a node are carried out independently of the transmissions of all other nodes.

We classify the nodes in two sets $N^{\prime}$ and $N \backslash N^{\prime}$, where $N^{\prime}=$ $\left\{i: \sum_{k \in K_{i} \cup\{i\}}\left|I_{k}\right|=\left|O_{i}\right|\right\}$. Consider a node $i \in N^{\prime}$. Refer to Fig. 1. Since $O_{i} \subseteq \cup_{k \in K_{i}} I_{k}$, it follows that: 1) $\left|I_{i}\right|=0$, i.e., $i$ does not have any in-neighbor, and 2) $\sum_{k \in K_{i}}\left|I_{k}\right|=\left|O_{i}\right|$, i.e., none of $i$ 's neighbors have any in-neighbor other than $i$. Therefore, node $i$ 's transmission does not reach the receiver of any other transmitting node. Moreover, by our assumption, $i$ 's transmission does not affect the transmission attempts by any other node. Therefore, node $i$ 's transmission can not reduce the throughput of any other node. Moreover, note that the throughput of node $i$ can not decrease as the transmission rate of node $i$ increases. Since the global fairness objective is an increasing (more specifically, logarithmic, as explained later) function of the throughputs, it follows that to achieve the desired fairness objective, node $i$ must transmit all the time. In other words, as soon as node $i$ finishes transmitting a packet, it begins transmitting the next packet. Since node $i$ must be involved in at least one active communication (by assumption), and $I_{i}=\phi$, it follows that $O_{i} \neq \phi$. Before transmitting each packet, node $i$ selects a destination $j \in O_{i}$ with probability $q_{(i, j)}$, where $\sum_{j \in O_{i}} q_{(i, j)}=1$. Let $\underline{q}=\left(q_{(i, j)}, i \in N^{\prime},(i, j) \in E\right)$ denote the vector of attempt probabilities on edges originating from the nodes in $N^{\prime}$.

Consider a node $i \in N \backslash N^{\prime}$. Refer to Fig. 1. Node $i$ 's transmissions interfere with transmissions to node $i$ or to any of node $i$ 's neighbors. Note that either node $i$ has some in-neighbor, or at least one of node $i$ 's neighbors has some in-neighbor. Therefore, for fair allocation of rates, $i$ should not always transmit packets. If $i$ has any out-neighbor, i.e., $O_{i} \neq \phi$, we assume that $i$ attempts to transmit as per a Poisson process with rate $\lambda_{i}$. If $O_{i}=\phi, i$ never transmits. Once $i$ attempts to transmit a packet, it selects a destination $j \in O_{i}$ with probability $\left(\lambda_{(i, j)} / \lambda_{i}\right)$, where $\sum_{j \in O_{i}} \lambda_{(i, j)}=\lambda_{i}$. Therefore, transmission attempts on $(i, j)$ are generated according to a Poisson process with rate $\lambda_{(i, j)}$. Thus, $\lambda_{(i, j)}$ can be viewed as the attempt rate in edge $(i, j), i \in N \backslash N^{\prime}$. If $i$ is already transmitting when the transmission attempt is generated (according to the Poisson process), then the new transmission attempt is ignored. Otherwise, $i$ transmits, even if it was receiving a packet at that instant. This assumption of "transmission takes precedence over reception" has been used by previous researchers as well [8]. Let $\underline{\lambda}=$ $\left(\lambda_{(i, j)},(i, j) \in E, i \in N \backslash N^{\prime}\right)$ denote the vector of attempt rates in edges originating from nodes in $N \backslash N^{\prime}$.

The following lemma shows how the throughput or the successful data rate on an edge depends on the attempt vector, $(\underline{q}, \underline{\lambda})$. In Lemma 2 and its proof, if $O_{k}=\phi$ for any $k \in N$, then the term $\lambda_{k}$ should be interpreted as zero.

Lemma 2: The throughput on edge $(i, j), x_{(i, j)}$, is given by (4) at the bottom of the page.

Tobagi et al. [8] computed the throughputs for unslotted Aloha protocol when each node attempts transmission according to a Poisson process, and the packet sizes are exponentially distributed.

The attempt vector, $\left(\underline{q}^{*}, \underline{\lambda}^{*}\right)$, that attains the proportionally fair rates, is given by

$$
\left(\underline{q}^{*}, \underline{\lambda}^{*}\right)=\arg \max _{\substack{q_{(i, j)} \geq 0, \sum_{j \in O_{i}} q_{(i, j)}=1, i \in N^{\prime} \\ \lambda_{(i, j)} \geq 0, i \in N \backslash N^{\prime},(i, j) \in E}} \sum_{(i, j) \in E} \log \left(x_{(i, j)}(\underline{q}, \underline{\lambda})\right)
$$

where $x_{(i, j)}$ are given in Lemma 2.

\section{B. Optimal Attempt Vector}

Theorem 3: The optimal attempt vector, $\left(q^{*}, \underline{\lambda}^{*}\right)$, as defined by (5), is given by $q_{(i, j)}^{*}=1 /\left|O_{i}\right|, i \in N^{\prime},(i, \bar{j}) \in E$, and $\lambda_{(i, j)}^{*}=$ $\lambda_{i}^{*} /\left|O_{i}\right|, i \in N \backslash N^{\prime},(i, j) \in E$, where

$$
\lambda_{i}^{*}=\frac{\sqrt{1+\frac{\left|O_{i}\right|}{\sum_{k \in K_{i} \cup\{i\}}\left|I_{k}\right|-\left|O_{i}\right|}}-1}{T}, \quad i \in N \backslash N^{\prime} .
$$

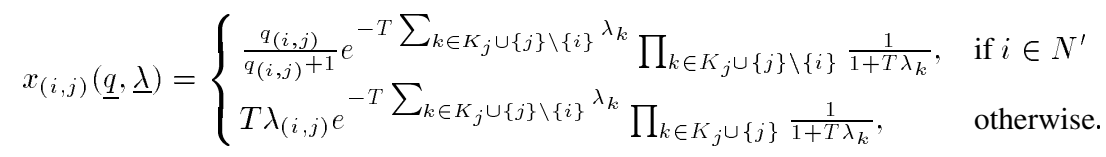


Consider a node $i \in N^{\prime}$. Recall that $O_{i} \neq \phi$. Clearly, $q_{(i, j)}^{*} \geq$ $0, q_{(i, j)}^{*} \leq 1$, and $\sum_{j \in O_{i}} q_{(i, j)}^{*}=1$. Consider a node $i \in N \backslash N^{\prime}$. Since $\sum_{k \in K_{i} \cup\{i\}}\left|I_{k}\right| \neq\left|O_{i}\right|, \sum_{k \in K_{i} \cup\{i\}}\left|I_{k}\right|>\left|O_{i}\right|$, as $O_{i} \subseteq \cup_{k \in K_{i} \cup\{i\}}\left|I_{k}\right|$, for all $i$. Thus, the optimal attempt vector is well defined.

Similar to the slotted Aloha case, a node can set its attempt vector optimally if it knows the number of its in-neighbors and out-neighbors, and the number of in-neighbors of its neighbors. It can obtain this knowledge by exchanging information with its neighbors.

Now, consider the special case in which all nodes are in each other's transmission range, and therefore, at most one node can send data successfully at any time. Let $n$ nodes send packets to a single destination node. Here, $N^{\prime}=\phi$. From Theorem 3, the optimal attempt rates in each of the $n$ edges is $(1 / T)(\sqrt{1+1 /(n-1)}-1) \approx(1 / 2 T(n-1))$, for large $n$. Now, from Lemma 2 , the system throughput is $(n / 2 \sqrt{e}(n-1)) \times\left(1 /(1+(1 / 2(n-1)))^{n}\right)$. As $n$ approaches $\infty$, the system throughput for proportionally fair rates becomes $(1 / 2 e) \approx 18 \%$, which equals the maximum system throughput attained for any choice of attempt rates in unslotted Aloha in this network [1].

\section{APPENDIX}

Proof of Theorem 1: Let $U(\underline{p})=\max _{0 \leq p_{e} \leq 1} \sum_{e \in E} \log$ $\left(x_{e}(\underline{p})\right)$. Then

$$
\begin{aligned}
U(\underline{p})=\sum_{(i, j) \in E}\left(\log \left(p_{(i, j)}\right)+\log \left(1-P_{j}\right)\right. & \\
& \left.+\sum_{k \in K_{j} \backslash\{i\}} \log \left(1-P_{k}\right)\right) .
\end{aligned}
$$

Note that $\log \left(p_{(i, j)}\right)$ is a strictly concave function of $p_{(i, j)}$. Also, for any $w \in N, \log \left(1-P_{w}\right)=\log \left(1-\sum_{k \in O_{w}} p_{(w, k)}\right)$ is a strictly concave function of $\left(p_{(w, k)}, k \in O_{w}\right)$. Thus, $U(\underline{p})$ is a strictly concave function of $p$. Therefore, $U(p)$ has a unique global maximum, which is obtained at $\underline{p}^{*}=\left(p_{e}^{*}, e \in E\right)$, where, $\left.\nabla U(\underline{p})\right|_{\underline{p}^{*}}=0$. Using $\left.\left(\partial U / \partial p_{(i, j)}\right)\right|_{\underline{p}^{*}}=\overline{0}, P_{w}=\sum_{k \in O_{w}} p_{(w, k)}$ for all $\bar{w} \in N,(6)$, and some algebraic manipulation, we obtain

$$
p_{(i, j)}^{*}=\frac{1-P_{i}^{*}}{\left|I_{i}\right|+\sum_{k \in K_{i}}\left|I_{k}\right|-\left|O_{i}\right|} \quad \forall(i, j) \in E
$$

where $P_{i}^{*}=\sum_{j \in O_{i}} p_{(i, j)}^{*}$. Select any $i \in N$. From (7), it follows that $p_{(i, j)}^{*}=P_{i}^{*} /\left|O_{i}\right| \forall j \in O_{i}$. Now, using (7), we obtain: $P_{i}^{*} /\left(1-P_{i}^{*}\right)=$ $\left|O_{i}\right| /\left(\left|I_{i}\right|+\sum_{k \in K_{i}}\left|I_{k}\right|-\left|O_{i}\right|\right)$. Thus, (3) follows.

Proof of Lemma 2: Consider an edge $(i, j) \in E$. A transmission attempt for edge $(i, j)$ generated at time instant $t$ leads to a successful packet transmission if: 1) node $i$ is not already transmitting at time $t$, and 2) none of the nodes $k \in K_{j} \cup\{j\} \backslash\{i\}$ transmit during the interval $[t, t+T]$. Condition 1 ) is necessary because if node $i$ is already transmitting at time $t$, then it ignores the newly generated transmission attempt. Since transmission takes precedence over reception, $i$ 's transmission is independent of any other transmission in the network; note that the success of a transmission depends on other transmissions though.

Consider a node $i \in N \backslash N^{\prime}$. We show that the probability that $i$ is not transmitting at an arbitrary time $t, P_{i}^{(1)}$, is equal to $\left(1 / \lambda_{i} T+1\right)$.
Recall that $i$ attempts to transmit according to a Poisson process with rate $\lambda_{i}$. Node $i$ 's packet transmissions can be modeled as a renewal process [9]. The renewal epochs are the epochs of $i$ 's transmission attempts that lead to packet transmissions; the transmissions may or may not collide with other transmissions. Recall that if $i$ is already transmitting at the epoch of a transmission attempt, it ignores the attempt. The time interval between two renewal epochs is the sum of the duration of a transmission and the subsequent wait time before a new attempt is generated. Using the memoryless property of exponential interattempt times, the expected time interval between two renewal epochs is $T+1 / \lambda_{i}$. Node $i$ transmits for the first $T$ units of time in each renewal period. From renewal-reward theorem [9], the probability that $i$ is transmitting at an arbitrary time $t$ is $T /\left(T+1 / \lambda_{i}\right)$. Thus, $P_{i}^{(1)}=1 /\left(\lambda_{i} T+1\right)$.

Note that for each $(i, j) \in E, K_{j} \cup\{j\} \backslash\{i\} \subseteq N \backslash N^{\prime}$. This can be inferred from the observation that no transmission from a node in $N^{\prime}$ collides with a transmission from another node, but transmission from nodes in $K_{j} \cup\{j\} \backslash\{i\}$ collides with a transmission on $(i, j)$.

Consider an edge $(i, j) \in E$. Let $P_{(i, j)}^{(2)}=P\left(\right.$ nodes $k \in K_{j} \cup\{j\} \backslash$ $\{i\}$ do not transmit in interval $(t, t+T))$. Since $K_{j} \cup\{j\} \subseteq N \backslash N^{\prime}$, each node $k \in K_{j} \cup\{j\} \backslash\{i\}$ attempts transmission as per a Poisson process. Now

$$
\begin{aligned}
P_{(i, j)}^{(2)}= & P\left(\text { none of the nodes } k \in K_{j} \cup\{j\} \backslash\{i\}\right. \\
& \text { attempt to transmit during }(\mathrm{t}, \mathrm{t}+\mathrm{T}) \mid \\
& \text { none of the nodes } k \in K_{j} \cup\{j\} \backslash\{i\} \\
& \text { are transmitting at } \mathrm{t}) \\
\times & P\left(\text { none of the nodes } k \in K_{j} \cup\{j\} \backslash\{i\}\right. \\
& \text { are transmitting at } \mathrm{t}) \\
= & e^{-T \sum_{k \in K_{j} \cup\{j\} \backslash\{i\}}{ }^{\lambda_{k}} \times \prod_{k \in K_{j} \cup\{j\} \backslash\{i\}} P_{k}^{(1)}} \\
= & e^{-T \sum_{k \in K_{j} \cup\{j\} \backslash\{i\}} \lambda_{k}} \times \prod_{k \in K_{j} \cup\{j\} \backslash\{i\}} \frac{1}{1+T \lambda_{k}} .
\end{aligned}
$$

Now, we compute the expression for the throughput on edge $(i, j)$ where $i \in N^{\prime}$. Recall that $i$ always transmits a packet. For each transmission, $i$ selects destination $j$ with probability $q_{(i, j)}$. Transmissions on $(i, j)$ can be modeled as a renewal process [9]. The renewal epochs are the epochs of the start of transmissions on $(i, j)$. The time interval between two renewal epochs is the sum of the duration of a transmission and the subsequent wait time before a new transmission on $(i, j)$. The first term equals $T$, and the second term equals $Z T$, where $Z$ is a geometrically distributed random variable with success probability $q_{(i, j)}$. Thus, the expected time interval between two renewal epochs is $T+T / q_{(i, j)}$. Node $i$ transmits for $T$ units of time on edge $(i, j)$ at the start of each renewal period. The transmission is successful if no node in $K_{j} \cup\{j\} \backslash\{i\}$ transmits during the transmission on $(i, j)$. Thus, $i$ 's average duration of successful transmission in a renewal interval is $T \times P_{(i, j)}^{(2)}$. From the renewal-reward theorem [9], the throughput on edge $(i, j)$ is $T \times P_{(i, j)}^{(2)} /\left(T+T / q_{(i, j)}\right)$. The expression for the throughput on edge $(i, j)$ follows using the expression for $P_{(i, j)}^{(2)}$.

Now, calculate the throughput on edge $(i, j)$, where $i \in N \backslash N^{\prime}$. Recall that $(i, j)$ attempts to transmit according to a Poisson process with rate $\lambda_{(i, j)}$. Now, using PASTA, the throughput in edge $(i, j)$ is $x_{(i, j)}=T \lambda_{(i, j)} \times P_{i}^{(1)} \times P_{(i, j)}^{(2)}$. The expression for the throughput in edge $(i, j)$ follows using the expressions for $P_{i}^{(1)}$ and $P_{(i, j)}^{(2)}$. 
Proof of Theorem 3: Using Lemma 2, the objective function of (5), $V(\underline{q}, \underline{\lambda})$ can be written as $V(\underline{q}, \underline{\lambda})=V_{1}(\underline{q})+V_{2}(\underline{\lambda})$, where $V_{1}(\underline{q})$ and $V_{2}(\underline{\lambda})$ are defined as

$$
\begin{aligned}
V_{1}(\underline{q})= & \sum_{i \in N^{\prime}} \sum_{j \in O_{i}} \log \frac{q_{(i, j)}}{1+q_{(i, j)}} \\
V_{2}(\underline{\lambda})= & \sum_{i \in N^{\prime} N^{\prime}}\left(\left(\sum_{j \in O_{i}} \log \left(T \lambda_{(i, j)}\right)\right)\right. \\
& -T \lambda_{i}\left(\left(\sum_{k \in K_{i} \cup\{i\}}\left|I_{k}\right|\right)-\left|O_{i}\right|\right) \\
& \left.-\left(\sum_{k \in K_{i} \cup\{i\}}\left|I_{k}\right|\right) \log \left(1+T \lambda_{i}\right)\right) .
\end{aligned}
$$

Here, $V_{1}(\underline{q})$ and $V_{2}(\underline{\lambda})$ consist of the terms in $V(\underline{q}, \underline{\lambda})$ that depend on $\underline{q}$ and $\underline{\lambda}$ respectively. It is easy to show that under the constraints $\sum_{j \in O_{i}} q_{(i, j)}=1, q_{(i, j)} \geq 0, i \in N^{\prime},(i, j) \in E, V_{1}(\underline{q})$ is maximized when $q_{(i, j)}=q_{(i, k)}$ for each $j, k \in O_{i}$ and each $i \in N^{\prime}$. Since $\sum_{j \in O_{i}} q_{(i, j)}=1$, it follows that $q_{(i, j)}^{*}=1 /\left|O_{i}\right|$.

Now, let us consider $V_{2}(\underline{\lambda})$, and see how it can be maximized over $\lambda_{(i, j)} \geq 0$. Note that $V_{2}(\underline{\lambda})$ is not concave over $\underline{\lambda} \geq \underline{0}$. Let $\underline{\lambda}^{*}$ be a local optimum of $V_{2}(\underline{\lambda})$. Then, $\left.\nabla V_{2}(\underline{\lambda})\right|_{\lambda^{*}}=0$. Consider any $i \in N \backslash N^{\prime}$. Then, for any $j \in O_{i}$, by setting $\left.\left(\partial V_{2} / \partial \lambda_{(i, j)}\right)\right|_{\underline{\lambda}^{*}}=0$ in (9), we obtain

$$
T \lambda_{(i, j)}^{*}=\frac{1}{\frac{\sum_{k \in K_{i} \cup\{i\}}\left|I_{k}\right|}{1+T \lambda_{i}^{*}}+\sum_{k \in K_{i} \cup\{i\}}\left|I_{k}\right|-\left|O_{i}\right|}
$$

where $\lambda_{i}^{*}=\sum_{j \in O_{i}} \lambda_{(i, j)}^{*}$. From (10), it follows that $\lambda_{(i, j)}^{*}=\lambda_{i}^{*} /\left|O_{i}\right| \forall j \in O_{i}$. Now, using (10), we obtain $T \lambda_{i}^{*}=\left|O_{i}\right| /\left(\sum_{k \in K_{i} \cup\{i\}}\left|I_{k}\right| /\left(1+T \lambda_{i}^{*}\right)+\sum_{k \in K_{i} \cup\{i\}}\left|I_{k}\right|-\left|O_{i}\right|\right)$. Solving for $\lambda_{i}^{*}$ in this quadratic equation, we obtain $\left.\lambda_{i}^{*}=(1 / T) \sqrt{1+\left|O_{i}\right| /\left(\sum_{k \in K_{i} \cup\{i\}}\right)\left|I_{k}\right|-\left|O_{i}\right|}\right)-1$. Thus, $V_{2}(\underline{\lambda})$ has a unique local optimum, given by $\underline{\lambda}^{*}$. By computing $\left.\nabla^{2} V_{2}(\underline{\lambda})\right|_{\lambda^{*}}$, it can be verified that $\underline{\lambda}^{*}$ is a local maximum. Now, $V_{2}(\underline{\lambda})$ approaches $-\infty$ at the boundaries of the region, i.e., when any $\lambda_{(i, j)}$ approaches 0 or $\infty$. Therefore, the global maximum of $V_{2}(\underline{\lambda})$ over $\underline{\lambda} \geq \underline{0}$ is attained at $\underline{\lambda}^{*}$. The result follows.

\section{REFERENCES}

[1] D. Bertsekas and R. Gallagher, Data Networks. Upper Saddle River, NJ: Prentice-Hall, 1992.

[2] F. P. Kelly, "Charging and rate control for elastic traffic," Eur. Trans. Telecommun., vol. 8, no. 1, pp. 33-37, 1997.

[3] F. Kelly, A. Maulloo, and D. Tan, "Rate control for communication networks: Shadow prices, proportional fairness, and stability," J. Oper. Res. Soc., vol. 49, no. 3, pp. 237-252, 1998.

[4] S. Low and D. E. Lapsley, "Optimization flow control, I: Basic algorithm and convergence," IEEE/ACM Trans. Networking, vol. 7, pp. 861-874, Dec. 1999.

[5] T. Nandagopal, T.-E. Kim, X. Gao, and V. Bharghavan, "Achieving MAC layer fairness in wireless packet networks," in Proc. ACM/IEEE MobiCom'00, Boston, MA, Aug. 2000, pp. 87-98.

[6] T. Ozugur, M. Naghshineh, P. Kermani, C. M. Olsen, B. Rezvani, and J. A. Copeland, "Balanced media access methods for wireless networks," in Proc. ACM/IEEE MobiCom'98, Dallas, TX, Oct. 1998.
[7] L. Tassiulas and S. Sarkar, "Maxmin fair scheduling in wireless networks," in Proc. Infocom. 2002, New York, NY, June 2002, pp. 763-772.

[8] F. A. Tobagi, "Modeling and performance analysis of multihop packet radio networks," Proc. IEEE, vol. 75, pp. 135-155, Jan. 1987.

[9] R. Wolff, Stochastic Modeling and the Theory of Queues. Upper Saddle River, NJ: Prentice-Hall, 1989. 